Nota Científica

(Short communication)

\title{
REGISTRO RECIENTE DE LA MARTUCHA (POTOS FLAVUS) PARA LA RESERVA DE LA BIOSFERA BARRANCA DE METZTITLÁN Y EL ESTADO DE HIDALGO, MÉXICO
}

\author{
RECENTE RECORD OF KINKAJOU (POTOS FLAVUS) IN THE BIOSPHERE RESERVE \\ BARRANCA OF METZTITLAN AND HIDALGO STATE, MEXICO
}

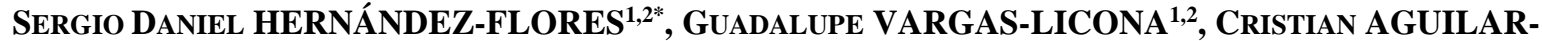 MIGUEL ${ }^{1}$, ALEJANDRO GARCÍA-BECERRA ${ }^{1}$, MARÍA DEL CARMEN GARCÍA-CHÁVEZ ${ }^{1}$}

\author{
${ }^{1}$ Naturaleza en Movimiento Ozomatli A.C. Constitución S/N, 43300, Atotonilco el Grande, Hidalgo. \\ ${ }^{2}$ Universidad Autónoma del Estado de Hidalgo, Escuela Preparatoria Número 1, Avenida Juárez 1100, Constitución, 42080, \\ Pachuca Hidalgo. \\ <danielhf83@hotmail.com>; <luvalicona@gmail.com>; <bioaguilar02@gmail.com>; <alejandrogarciabecerra@ hotmail.com>; \\ <carmen.lob.gch@gmail.com> \\ *Autor de correspondencia: <danielhf83@hotmail.com>
}

Recibido: 04/04/2018; aceptado: 17/10/2018; publicado en línea: 10/12/2018

Editor responsable: Vinicio Sosa

Hernández-Flores, S. D., Vargas-Licona, G., Aguilar-Miguel, C., García-Becerra, A., García-Chávez, M. C. (2018) Registro reciente de la martucha (Potos flavus) para la Reserva de la Biosfera Barranca de Metztitlán y el estado de Hidalgo, México. Acta Zoológica Mexicana (nueva serie), 34, 1-5. https://doi.org/10.21829/azm.2018.3412158

RESUMEN. Con base en el estudio de especies realizado en las áreas protegidas dentro del estado de Hidalgo, identificamos un espécimen de martucha cazado en la porción sur del municipio de Zacualtipan. El espécimen fue cazado durante la primera semana de mayo de 2016. Confirmamos la presencia reciente de la especie en el estado de Hidalgo y en la Reserva de la Biosfera Barranca de Metztitlán. Por lo tanto, esperamos realizar más estudios en esta área que brinden una mejor comprensión de este taxón en México y su asociación con la pérdida de vegetación.

Hernández-Flores, S. D., Vargas-Licona, G., Aguilar-Miguel, C., García-Becerra, A., García-Chávez, M. C. (2018) Recente record of kinkajou (Potos flavus) in the Biosphere Reserve Barranca of Metztitlan and Hidalgo state, Mexico. Acta Zoológica Mexicana (nueva serie), 34, 1-5. https://doi.org/10.21829/azm.2018.3412158

\begin{abstract}
Based on the species survey conducted in the protected areas within Hidalgo state, we identified a hunted specimen of kinkajou in the southern portion of the municipality of Zacualtipan. The specimen was hunted during the first week of May 2016. We confirm the recent presence of the species in Hidalgo state and the Biosphere Reserve Barranca of Metztitlan. Hence, we expect to conduct more studies in this area that provide a better understanding of this taxon in Mexico and its association with vegetation loss.
\end{abstract}


La martucha o mico de noche es un carnívoro prociónido de tamaño medio, pesando entre 1.4 y $4.6 \mathrm{~kg}$. (Figueroa \& Arita, 2005). Entre los miembros de la familia Procyonidae, es la especie arborícola más especializada: sus articulaciones en los tobillos le permiten rotar hacia atrás y descender de los árboles con la cabeza hacia abajo, apoyándose en su cola prensil que funciona como una quinta extremidad (Ford \& Hoffmann, 1988). Habita principalmente bosques tropicales con una producción constante de frutos todo el año. En algunos lugares se ha reportado que del 90 al 99\% de su dieta está constituida por frutos. Los ecosistemas que habita incluyen el bosque tropical lluvioso, bosque de niebla, bosques secos y vegetación ribereña. Se ha reportado desde el nivel del mar hasta los 2,500 msnm. La martucha utiliza todos los estratos arbóreos y solo en raras ocasiones se la ha observado en el suelo del bosque (Ford \& Hoffman, 1988; Kays, 2009). Sus hábitos son completamente nocturnos y durante el día reducen su tasa metabólica mientras duermen en árboles huecos o en nichos formados en el dosel de los árboles (Figueroa \& Arita, 2005; Kays, 2009).

Es una especie considerada de preocupación menor (least concern) en la Lista Roja de la IUCN, sin embargo, su tendencia poblacional se considera en descenso (Helgen et al., 2016). Con base en la legislación mexicana (NOM-059-ECOL-2010), su estatus es considerado bajo protección especial (SEMARNAT, 2010), entre otras razones debido a que habita algunos de los ecosistemas más amenazados en México, como el bosque mesófilo de montaña y el bosque tropical perennifolio (Figueroa \& Arita, 2005; CONABIO, 2010).

En México su rango de distribución va desde el sur de Tamaulipas hasta el sureste mexicano, incluyendo los estados de Yucatán, Quintana Roo, Tabasco, Veracruz, parte de San Luis Potosí y Tamaulipas, en el Golfo de México; también se ha registrado en Chiapas, Oaxaca, Guerrero y Michoacán del lado del Pacífico. En el centro de México existen registros aislados en Puebla e Hidalgo (Figueroa \& Arita, 2005; Ford \& Hoffman, 1988; Helgen et al., 2016; Hall 1981; Kays 2009; Sánchez-Rojas et al., 2016). Particularmente en el estado de Hidalgo, la escasez de registros no permite conocer el rango de distribución real, dado que aun cuando se ha reportado en numerosos trabajos, la mayoría de ellos se basan en un solo registro de 1970 (Jones et al., 1983; López-Wilchis \& López-Jardines, 1999; Ramírez-Pulido et al., 2000; Mejenes-López et al., 2010; Sánchez-Rojas et al., 2016).

El objetivo de la presente contribución es reportar la presencia de la martucha para el estado de Hidalgo como parte del monitoreo realizado en sus áreas naturales protegidas para identificar y preservar sus especies silvestres.

La zona se monitoreó mediante rastreo y trampas cámara, no obstante, el registro se fundamenta en un ejemplar cazado en el municipio de Zacualtipán (20.564569, -98.645656; 1,380 msnm) por una persona de la región, la primera semana de mayo del año 2016 (Fig. 1, 2). El sitio donde fue cazado el ejemplar corresponde a una cañada cubierta por vegetación ribereña con presencia de mezquites (Prosopis leviagata) y sauces (Salix humboldtiana). El árbol donde fue localizado el animal se encuentra justo en el cauce del río. La vegetación circundante corresponde a un matorral crasicaule con dominancia de Isolatocereus durmortieri, Myrtillocactus geometrizans y Cephalocereus senilis. No se identificó con certeza el sexo del animal dado que el ejemplar se revisó varios días después de ser cazado, cuando la piel fue curtida y solo se tomaron las medidas estándar: LT: (1020), C: (460), P: (80), O: (820). Pese a lo anterior se recuperó una fotografía del animal tomada al día siguiente de la captura (Fig. 2).

La importancia del hallazgo radica en que se confirma la presencia reciente de la martucha en el estado de Hidalgo, aún en zonas áridas y dentro de la Reserva de la Biosfera Barranca de Metztitlán. Previo a este ejemplar, existen solamente dos registros publicados para la especie en el estado de Hidalgo; el primero se fundamenta en un cráneo colectado por habitantes de la región de Zacualtipán en 1970 y reportado por Jones et al. (1983), mismo que se encuentra en la colección de Texas Museum (no. 24634), registro reportado en varios trabajos que han realizado una revisión de los mamíferos presentes en el estado 
de Hidalgo y ubicado $1.7 \mathrm{~km}$ al norte del presente hallazgo (López-Wilchis \& López-Jardines 1999; Ramírez-Pulido et al., 2000; Mejenes-López et al., 2010; Sánchez-Rojas et al., 2016). De igual forma el segundo registro es el de Aguilar et al. (2013) para la región de Tlanchinol, sin embargo, fue considerado con base en entrevistas realizadas a personas de la zona, aproximadamente $46.8 \mathrm{~km}$ al norte del registro reportado en la presente, pues no es posible ubicarlo con certeza ya que en ese trabajo no se obtuvo ningún registro puntual de la especie.

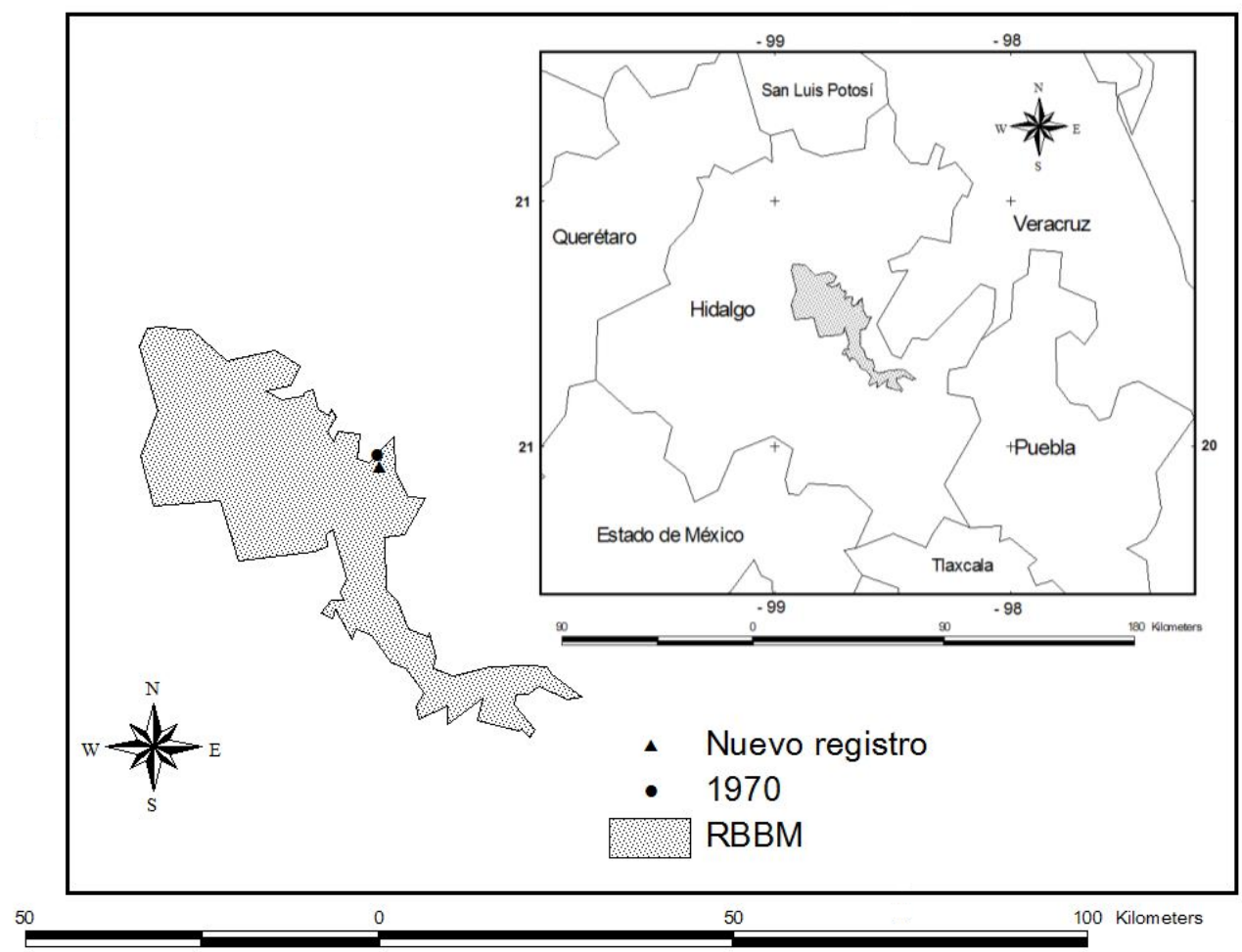

Figura 1. Ubicación del nuevo registro para la martucha (Potos flavus) en la Reserva de la Biosfera Barranca de Metztitlán y el estado de Hidalgo (se indica con un triángulo). Además, se muestra el único registro confirmado previamente que data de 1970 (con un círculo).

Finalmente, a partir del presente hallazgo se espera plantear nuevos estudios sobre la martucha en la zona, ya que en México solo se conoce como parte de inventarios y registros aislados (Figueroa \& Arita 2005; Ford \& Hoffman, 1988; Gómez-Nísino, 2006; Hall, 1981; Jones et al., 1983). Se ha planteado que puede desempeñar un papel muy importante como dispersor de semillas e incluso como polinizador en los ecosistemas que habita (Aranda, 2012), aunque actualmente apenas existe la información requerida para determinar su distribución (Figueroa \& Arita, 2005). El desconocimiento sobre la especie se agrava aún más si consideramos su sensibilidad a la deforestación y la pérdida de cobertura vegetal, al ser una especie arborícola muy especializada, hecho que hace emergente su estudio a corto plazo (Ford \& Hoffman, 1988; Helgen et al., 2016; Kays, 2009). 


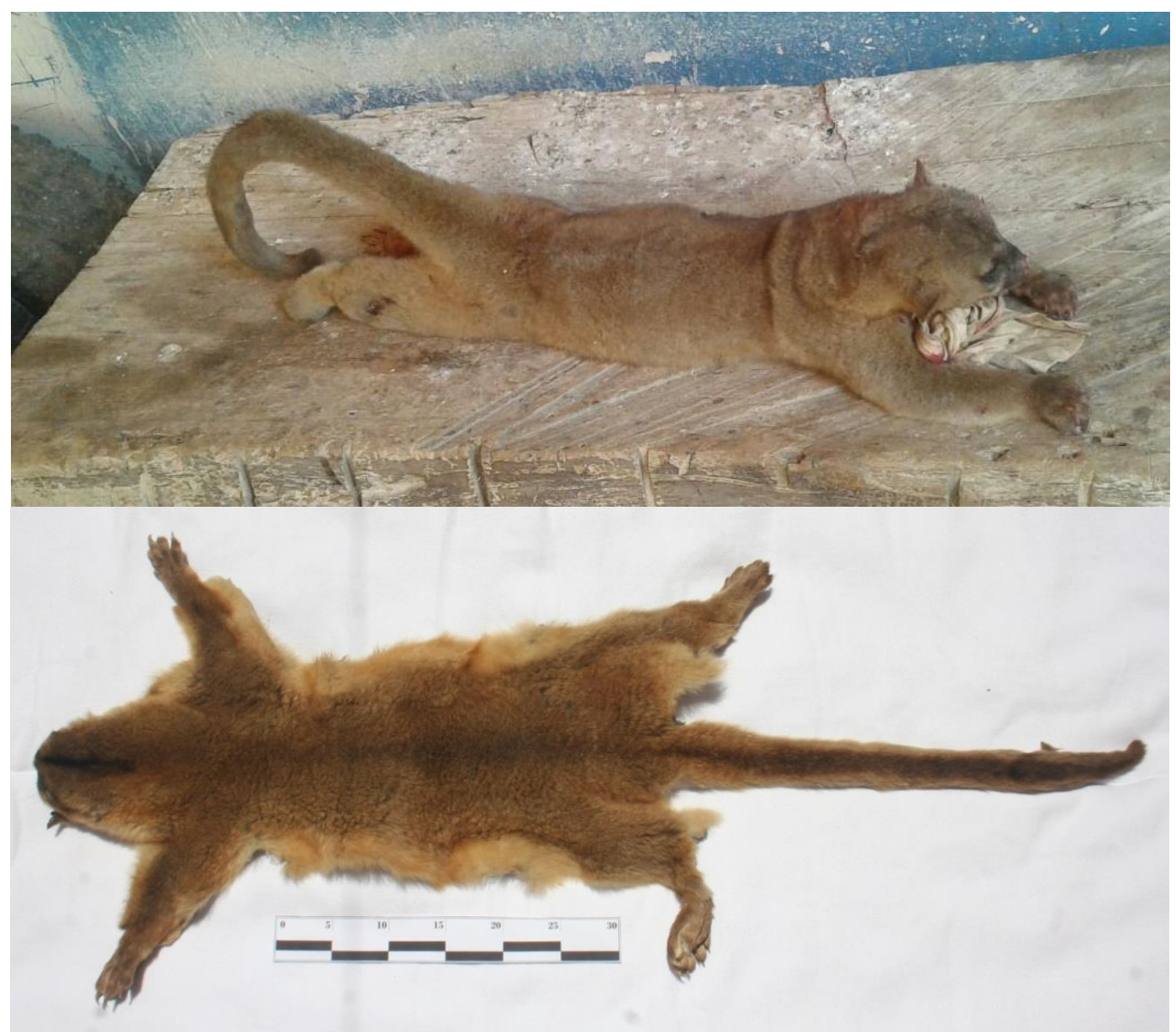

Figura 2. Muestra al ejemplar al día siguiente de ser cazado (parte superior) y se observa la piel curtida del mismo (parte inferior).

AGRADECIMIENTOS. Esta investigación fue posible gracias al valioso apoyo de Guillermo Sánchez y los pobladores de la región. A la M. en C. Margarita Medina se agradece por su valiosa ayuda en la traducción del resumen. Finalmente, a los revisores anónimos.

\section{LITERATURA CITADA}

Aguilar-López, M., Rojas Martínez, A. E., Cornejo-Latorre, C., Vite-Silva, V. D., Ruano-Escalante, I. R. (2013) Lista taxonómica y estructura del ensamblaje de los mamíferos terrestres del municipio de Tlanchinol, Hidalgo, México. Mastozoología Neotropical, 20, 229-242.

Aranda, M. (2012) Manual para el rastreo de mamíferos silvestres de México. CONABIO. México, D.F. $255 \mathrm{pp}$.

CONABIO (2010) El Bosque Mesófilo de Montaña en México: Amenazas y Oportunidades para su Conservación y Manejo Sostenible. CONABIO. México D.F.

Figueroa, F., Arita, H. T. (2005) Potos flavus, pp. 405-408 In: Ceballos, G., Oliva, G. (Eds.). Los mamíferos silvestres de México. CONABIO, Fondo de Cultura Económica, México, D.F.

Ford, L. S., Hoffmann, R. S. (1988) Potos flavus. Mammalian Species, 321, 1-9.

Gómez-Nísino, A. (2006) Ficha técnica de Potos flavus. In: Medellín, R. (compilador). Los mamíferos mexicanos en riesgo de extinción según el PROY-NOM-059-ECOL-2000. Instituto de Ecología, 
Universidad Nacional Autónoma de México. Bases de datos SNIB-CONABIO. Proyecto No. W005. México, D.F. Disponible en:

http://www.conabio.gob.mx/conocimiento/ise/fichasnom/Potosflavus00.pdf (acceso en octubre de 2017)

Hall, R. E. (1981) The Mammals of North America. John Wiley and Sons. New York, EE.UU.

Helgen, K., Kays, R., Schipper, J. (2016) Potos flavus. The IUCN Red List of Threatened Species 2016. Disponible en: http://dx.doi.org/10.2305/IUCN.UK.2016 1.RLTS.T41679A45215631.en. (acceso en octubre de 2017).

Jones, J. K., Carter, D. C., Webster, W. M. (1983) Records of mammals from Hidalgo, Mexico. The Southwestern Naturalist, 28, 378-380.

Kays, R. (2009) Family Procyonidae (Racoons), pp. 504-531. In: Wilson, D. E., Mittermeier, R.A. (Eds.). Handbook of the Mammals of the World, v. 1. Carnivores Lynx Editions, Barcelona.

López-Wilchis, R., López-Jardines, J. (1999) Los Mamíferos de México Depositados en Colecciones de Estados Unidos y Canadá. Vol. 2. Universidad Autónoma Metropolitana. Unidad Iztapalapa. México, D.F.

Mejenes-López, S. de M., Hernández, M., Barragán, J., Pacheco, J. (2010) Los mamíferos en el estado de Hidalgo. Therya, 3, 161-188.

Ramírez-Pulido J., Castro, A., Armella, M., Salame, A. (2000) Bibliografía reciente de los mamíferos de México: 1994-2000. Universidad Autónoma Metropolitana, Unidad Iztapalapa. México D.F.

Sánchez-Rojas G., Hernández Flores, S. D., Castillo-Cerón, J., Mejenes-López, S., Aguilar-López, M., Bravo-Cadena, J., García-Becerra, A., García-Morales, R., Hernández-Silva, D. (2016) Riqueza, composición y conservación de los mamíferos del estado de Hidalgo, México, pp. 281310 In: Briones-Salas, M., Hortelano-Moncada, Y., Magaña-Cota, G., Sánchez-Rojas, G., SosaEscalante, J. E. (Eds.). Riqueza y Conservación de los Mamíferos en México a Nivel Estatal, Instituto de Biología, UNAM, Asociación Mexicana de Mastozoología A.C. y Universidad de Guanajuato. México, D.F.

SEMARNAT (2010) Norma Oficial Mexicana NOM-059-ECOL-2010, Protección ambiental-Especies nativas de México de flora y fauna silvestres-Categorías de riesgo y especificaciones para su inclusión, exclusión o cambio-Lista de especies en riesgo. México, D.F. 